\title{
The relationship between the magnetosphere and magnetospheric/auroral substorms
}

\author{
S.-I. Akasofu \\ International Arctic Research Center, University of Alaska Fairbanks, AK 99775 Fairbanks, USA
}

Correspondence to: S.-I. Akasofu (sakasofu@iarc.uaf.edu)

Received: 25 October 2012 - Revised: 19 December 2012 - Accepted: 28 January 2013 - Published: 4 March 2013

\begin{abstract}
On the basis of auroral and polar magnetic substorm studies, the relationship between the solar windmagnetosphere dynamo (the DD dynamo) current and the substorm dynamo (the UL dynamo) current is studied. The characteristics of both the DD and UL currents reveal why auroral substorms consist of the three distinct phases after the input power $\varepsilon$ is increased above $10^{18} \mathrm{erg} \mathrm{s}^{-1}$. (a) The growth phase; the magnetosphere can accumulate magnetic energy for auroral substorms, when the ionosphere cannot dissipate the power before the expansion phase. (b) The expansion phase; the magnetosphere releases the accumulated magnetic energy during the growth phase in a pulse-like manner in a few hours, because it tries to stabilize itself when the accumulated energy reaches to about $10^{23} \mathrm{erg} \mathrm{s}^{-1}$. (c) The recovery phase; the magnetosphere becomes an ordinary dissipative system after the expansion phase, because the ionosphere becomes capable of dissipating the power with the rate of $10^{18} \sim 10^{19} \mathrm{erg} \mathrm{s}^{-1}$.
\end{abstract}

On the basis of the above conclusion, it is suggested that the magnetosphere accomplishes the pulse-like release process (resulting in spectacular auroral activities) by producing plasma instabilities in the current sheet, thus reducing the current. The resulting contraction of the magnetic field lines (expending the accumulated magnetic energy), together with break down of the "frozen-in" field condition at distances of less than $10 R_{\mathrm{E}}$, establishes the substorm dynamo that generates an earthward electric field (Lui and Kamide, 2003; Akasofu, 2011). It is this electric field which manifests as the expansion phase. A recent satellite observation at a distance of as close as $8.1 R_{\mathrm{E}}$ by Lui (2011) seems to support strongly the occurrence of the chain of processes suggested in the above.

It is hoped that although the concept presented here is very crude, it will serve in providing one way of studying the three phases of auroral substorms. In turn, a better understanding of auroral substorms will also be useful in studying the magnetosphere, because various auroral activities can be the visible guide for this endeavor.

Keywords. Magnetospheric physics (Storms and substorms)

\section{Introduction}

Auroral substorms and polar magnetic disturbances are different manifestations of electrical discharge currents which grow and decay during magnetospheric substorms; the aurora is the optical manifestation, while polar magnetic disturbaces are the magnetic manifestation. It so happened that the general pattern of both auroral substorms and the substorm current system was established coincidentally by Akasofu (1964) and Bostrom (1964) respectively in 1964; see Fig. 1. Their results have served as a general guide in studying both phenomena, and their general validity, as a first approximation, was confirmed for auroral substorms by Frank and Craven (1988) and for the current system by Akasofu (1992).

In this paper, we attempt to examine auroral and polar magnetic substorms together in terms of electrical discharge and circuit (Fig. 1), so that we must combine both the optical and the magnetic manifestations in order to learn about the whole discharge circuit.

In studying substorms, it is important to recognize that they have three distinct phases, the growth phase, the expansion phase and the recovery phase. In this regard, we might recall that Chapman (1918) established the present concept of geomagnetic storms, consisting of the storm sudden commencement, the initial phase, the main phase and 

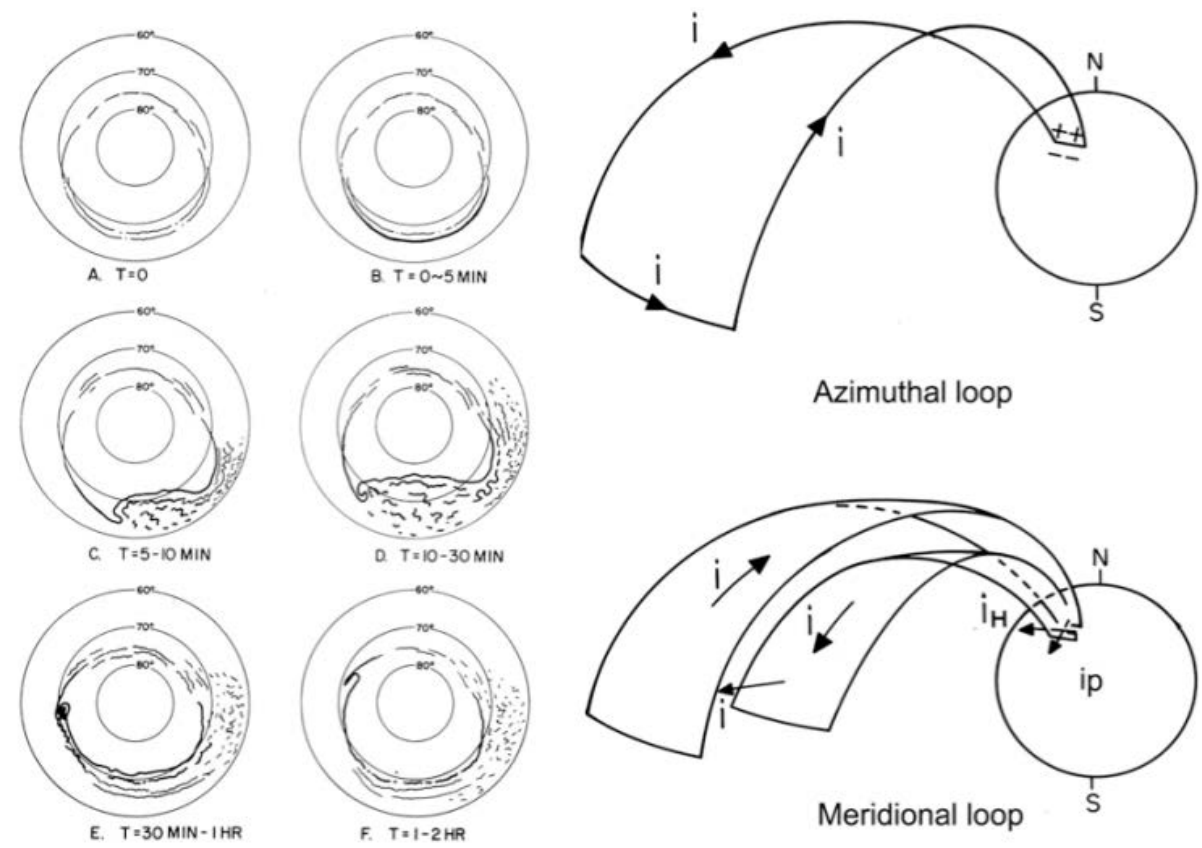

Azimuthal loop

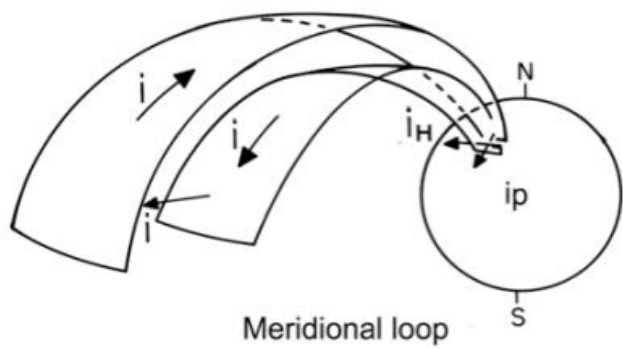

Fig. 1. Left: The pattern of the development of auroral substorms (Akasofu, 1964). Right: The substorm current system which consists of the meridional component and the azimuthal component (Bostrom, 1964).

the recovery phase. It was this morphological concept that has led the later generations to study each phase in terms of physics. Chapman interpreted the storm sudden commencement as an impact of isolated plasma clouds, compressing the earth's dipole field. The storm sudden commencement is now understood to be caused by the impact of the interplanetary shock wave, which propagates ahead of the advancing magnetic cloud (ICME). The impact effect is then propagated in the magnetosphere as the Alfvén waves before reaching the earth's surface. Chapman speculated that the main phase is caused by solar wind particles circulating around the earth, the ring current (Chapman and Ferrao, 1941). However, the true nature of the ring current had to wait until the discovery of the Van Allen belt and of $\mathrm{O}^{+}$ions.

In a similar way, by recognizing characteristics of each of the three phases, substorm studies are expected to make a new progress, since we are still at an early stage of understanding auroral/magnetospheric substorms. It is hoped that this paper will be able to serve in advancing this field.

\section{The DD/UL currents and the requirements for the UL dynamo}

Since a current system must be powered by a dynamo process, a study of auroral substorms and polar magnetic disturbances must find a dynamo which can satisfy requirements established by the relevant observations (cf. Akasofu, 2003).

1. Bostrom's current system (Fig. 1) requires only one electric field in the ionosphere for both the meridional component and the azimuthal component. It is an equatorward directed field that drives both the Pedersen current $(\boldsymbol{E} \times \boldsymbol{J}>0)$ and the Hall current $(\boldsymbol{E} \times \boldsymbol{J}=0)$; it is also the sheet current (carried by downward streaming electrons) in the meridional component that is directly responsible for an auroral arc.

2. The only part of the circuit in which $\boldsymbol{E} \times \boldsymbol{J}<0$, is located in the equatorial part of the meridional circuit. Thus, the electric field $\boldsymbol{E}$ must be located there and be directed earthward.

3. Thus, the dynamo should be able to generate an earthward electric field.

In order to find the substorm dynamo which can satisfy the three requirements above, it is necessary to identify and determine, first of all, the substorm (UL, unloading) current by separating it from the solar wind-magnetosphere (DD, directly driven) current; magnetometers record the field of the (DD + UL) current. Sun et al. (1998) succeeded in this separation process in terms of equivalent current lines (equivalent to electric potential lines and the $\boldsymbol{E} \times \boldsymbol{B}$ flow lines of ionospheric electrons). Based on the method of natural orthogonal components (the MNOC method), the DD current is denoted by Mode 1 (DD) and the UL current by Mode 2 (UL). This mathematical separation is possible because the UL current is a one-cell current, while the DD current is a two-cell current. The data based on the six International Meridian Chains (Kamide et al., 1982) were used for this purpose. Figure 2 shows the averaged DD and UL equivalent 


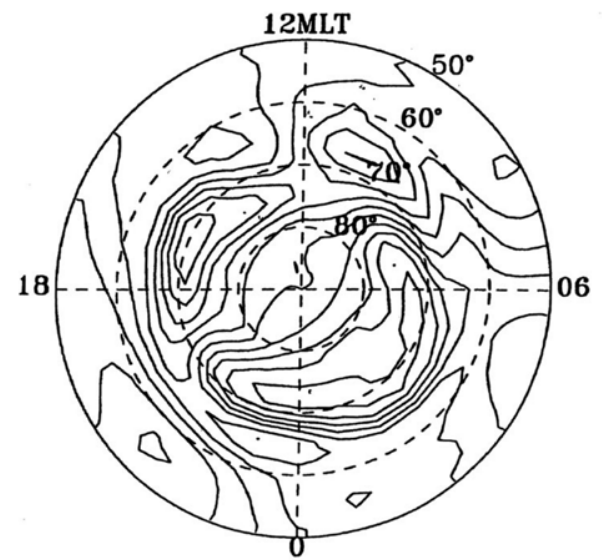

DIRECTLY DRIVEN COMPONENT

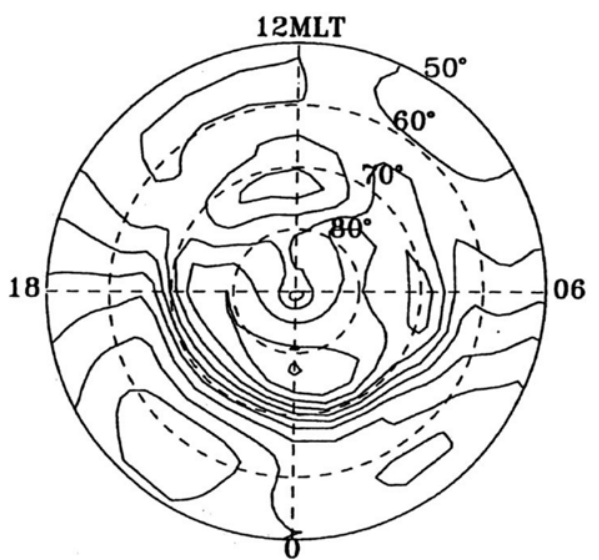

UNLOADING COMPONENT

Fig. 2. Left: The solar wind-magnetosphere DD (the two-cell) current. Right: The substorm UL (the one-cell) current; (Sun et al., 1998).

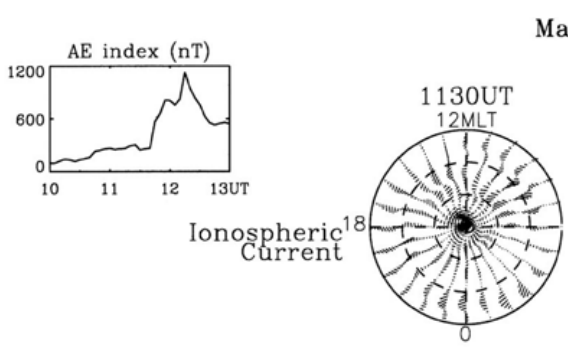

March 19, 1978
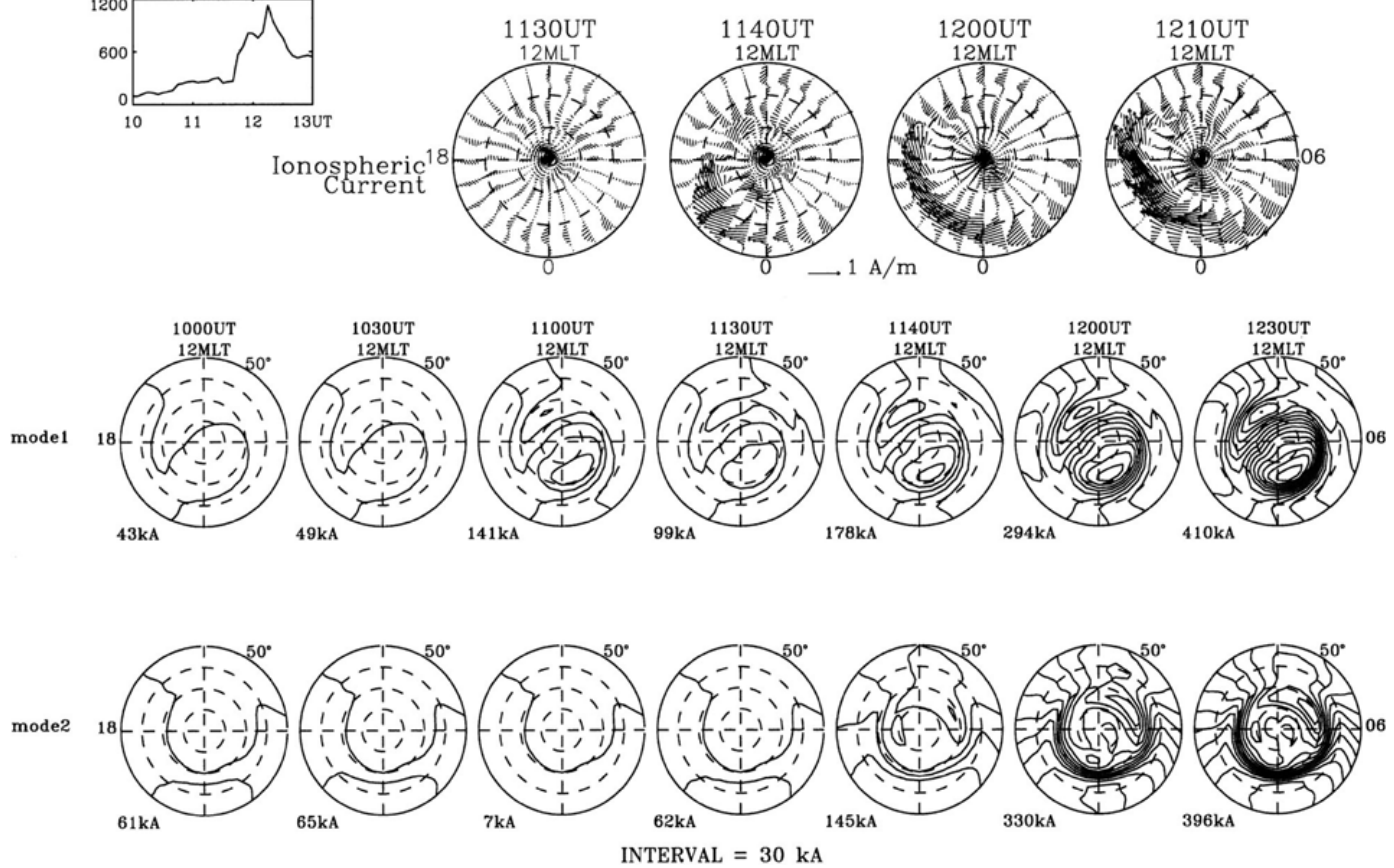

Fig. 3. From the top, the substorm current in the ionosphere, Mode 1 (DD) current and Mode 2 (UL) current (Sun et al., 1998). The AE index is shown on the upper left corner.

currents during the maximum epoch of substorms. It can be seen that DD current has a two-cell current pattern (greatly distorted from an ideal symmetric case), while the UL current has a one-current pattern.

The DD current pattern is very similar to the average flow pattern of electrons observed by the SuperDARN network (Bristow and Jensen, 2007). The UL current pattern shows the electron flow pattern (the Hall current) of the auroral electrojet, indicating also the presence of the equatorward elec- tric field in the dark sector (perpendicular to the Hall current lines and also to the equipotential lines).

In Fig. 3, the development of both DD and UL currents, together with the total ionospheric current, are shown for a substorm on 19 March 1978. It can be seen that the DD twocell current was clearly detected at about 11:00 UT by the MNOC method and then intensified after substorm onset at 10:40 UT; these facts are crucial in understanding the growth phase, as shown in the next section. A rapid growth of the westward electrojet (the UL current) can be seen at the time 


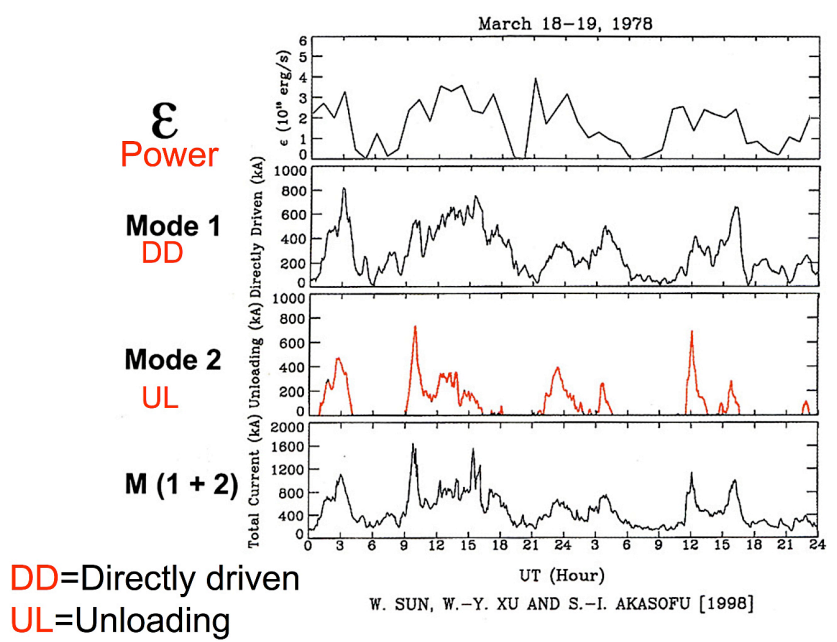

Fig. 4. From the top, the solar wind-magnetosphere dynamo power $\varepsilon$, the DD two-cell current intensity (Mode 1), the substorm (UL) current (Mode 2) and the combination of Mode 1 and Mode 2 (corresponding to an improved $\mathrm{AE}$ index, because the total current intensity is presented).

of substorm onset. The results shown in Fig. 3 may also serve as proof of the MNOC method in this application, since the results are in agreement with what we have known qualitatively in the past.

Figure 4 shows the power $\varepsilon(t)$, the $\mathrm{DD}(t)$ and $\mathrm{UL}(t)$ currents as a function of time in studying auroral substorms for two days in terms of electric circuit, 18 and 19 March 1978, although it is not possible to monitor the current in the current sheet (inferred to be proportional to the power $\varepsilon$ and the DD current). In the night side of the polar ionosphere, the conductivity $\sigma$ is very low during a quiet period, but increases roughly in proposition to the field-aligned current intensity $I$ because the field-aligned current ionizes the ionosphere, so that the Joule heat loss $\delta\left(=I^{2} / \sigma\right)$ becomes roughly proportional to $I$ (also expected to be roughly proportional to the UL current). Note also that the magnetosphere and the ionosphere respond clearly to the power $\varepsilon$ when it becomes above $10^{18} \mathrm{erg} \mathrm{s}^{-1}$ (cf. Akasofu, 1981).

The results shown in Figs. 3 and 4 will be discussed fully in the next section. Here, it is pointed out that the most important fact is that the Mode 2 (the substorm UL current) shows a pulse-like change in two typical substorms (one which began at about 09:00 UT on 18 March and the other which began at about 11:00 UT on 19 March), very different from the DD current, indicating that the substorm (UL) dynamo is distinctly different from the DD dynamo. In general, the duration of the pulses is about three hours. Further, at the peak of the pulse-like change, the UL current is greater than the DD current, indicating also that the UL current results from the accumulated energy, not simply an enhancement of the DD current. The substorms started at 21:00 UT on 18 March will be discussed in Sect. 4. Note also that the AE index monitors only the (DD + UL) current.

\section{The three phases of auroral substorms}

In order to interpret the results in Figs. 3 and 4, it is useful to introduce the concept of electrical circuit. Figure 5 shows a set of conceptual circuits for the three phases of auroral substorms, although it is a very crude one. The whole system is driven by the solar wind-magnetosphere (DD) dynamo. Bostrom's circuit (Fig. 1) is represented in the expansion phase circuit, indicated by "substorm (UL) dynamo".

In attempting to discuss the substorm circuit, we realize that the present physical interpretation of auroral substorms relies almost entirely on the concept of moving magnetic field lines. However, in as early as 1967, Alfvén (1967, p. 440) stated, "In some applications we can illustrate essential properties of the electromagnetic state of space either by depicting the magnetic field lines or by depicting the electric current lines. Almost always the first picture is used exclusively. It is important to note that in many cases the physical basis of the phenomena is better understood if the discussion is centered on the picture of the current lines." In this paper, this is the reason why the "current line" approach is adopted, hoping that the circuit concept might throw some light on our difficult problem from a different point of view.

Further, Alfvén (1977, p. 276) emphasized: "Hence, in order to understand the properties of a current-carrying plasma we must take account of the properties of the whole circuit in which the current flows."; the word "whole" is italicized by the author for emphasis. In fact, although the current in the current sheet, the field-aligned currents, and the ionospheric current are frequently mentioned in the literature, there has not been much discussion of the substorm current circuit as $a$ whole (as well as the three phases of substorms), and further the terms, such as dynamo, inductance $L$, and Joule heat dissipation are not very often mentioned in the recent literature. This is an indication that substorm studies are somewhat fragmented, concentrating specifically on studies of different parts of the current circuit. One way to synthesize these studies may be to introduce the concept of current circuit; it provides one of the most important quantities in substorm studies, magnetic energy $W=(1 / 2) J^{2} L$, which is the source energy of the expansion phase of auroral substorms.

\subsection{Growth phase}

It is generally agreed that the magnetosphere accumulates the substorm energy $W=J^{2} L$ during the growth phase. In many ordinary systems, the energy input rate $\varepsilon(t)$ is equal to the energy dissipation rate $\delta(t-\tau)$, where $\tau$ is the system delay. Such a system tends to dissipate input energy with a certain system delay, so that there occurs little accumulation of energy. 

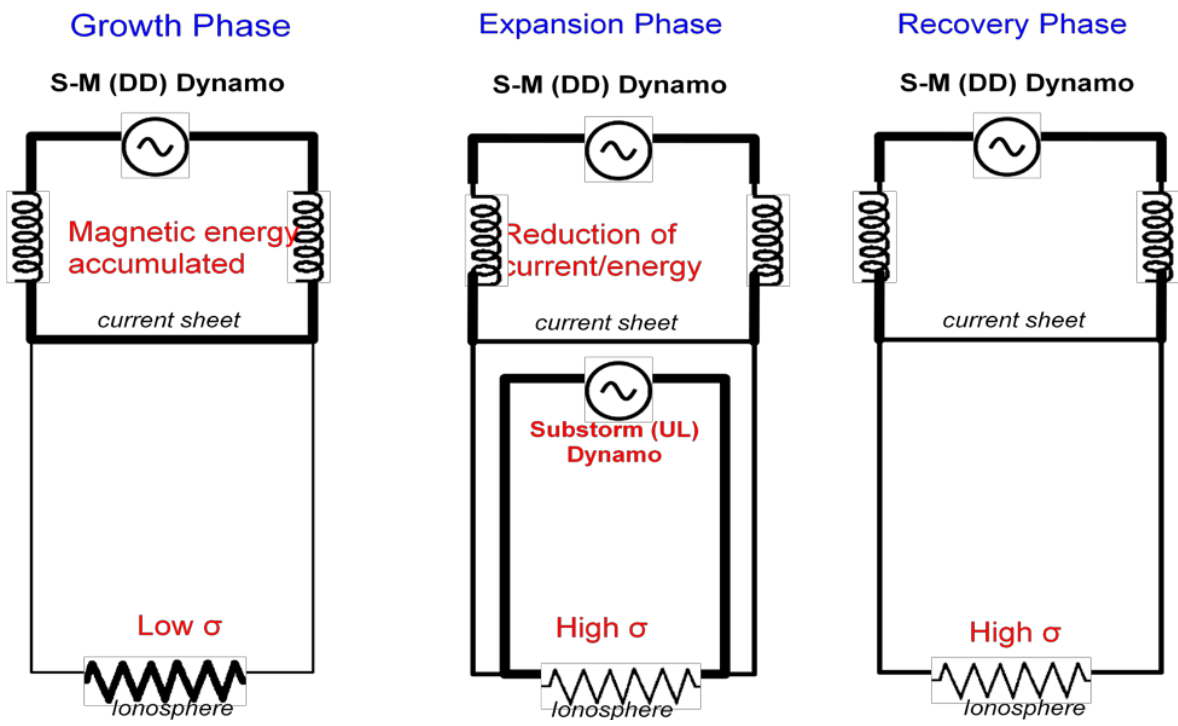

Fig. 5. The conceptual circuits for the three phases of auroral substorms.

The first question is then why the magnetosphere can accumulate the substorm energy $W$. It is suggested that when the ionosphere, the main sink of the input energy, is not conductive enough during the growth phase, it does not allow much current to flow there and cannot sufficiently dissipate the input energy produced by $\varepsilon$. In fact, we observed in Fig. 3 that only a weak DD current started to grow from a little before 11:00 UT and was intensified only after substorm onset at 11:40 UT. Therefore, this is an important indication that the dissipation in the ionosphere was very small until substorm onset at 11:40 UT. The growth phase in Fig. 5 shows a thin line between the dynamo and the ionosphere, representing this situation; a high resistance (a low conductivity) is shown as a thick resistivity sign.

Auroral changes during the growth phase is not well established yet; the equatorward half of the auroral oval appears to contract just prior to substorm onset (Akasofu et al., 2010). This phenomenon may be related to the thinning of the current sheet near the earthward end (Birn et al., 1998).

On the other hand, the current in the current sheet must be increased (perhaps in proportion to $\varepsilon$ ) during the growth phase, so that magnetic energy is accumulated. This feature is often expressed by stating that the dipole field lines in the dark side are "stretched" during the growth phase.

\subsection{Expansion phase}

The second question is why the release process of the accumulated energy is pulse-like (as Fig. 4 shows) and also why the auroral expansion phase lasts only about one hour or so. It is suggested that the magnetosphere tends to become unstable when the accumulated energy reaches about $W=5 \times 10^{22} \mathrm{erg} \mathrm{s}^{-1}$, a typical substorm energy $\left(\mathrm{J}=10^{7}\right.$ amperes and $L=64 \mathrm{H}$; Horton and Doxas, 1996). In restor- ing its stability, the current in the current sheet must be reduced by developing plasma instabilities in the current sheet. As a result, the accumulated magnetic energy is released.

The accumulated magnetic energy is exhausted in a few hours (inferring from the duration of the pulses), because a typical dissipation rate is about $10^{19} \mathrm{erg} \mathrm{s}^{-1}=10^{11} \mathrm{~W}$ at about the maximum epoch of substorms (Akasofu, 1985), and thus $W / \delta$ is about one hour or less in those cases. Both the quantities $W$ and the dissipation rate $\delta$ in individual substorms are expected to vary somewhat; the optical manifestation of the expansion phase appears to be shorter than the duration of the current pulses. It may also be noted that the time constant of the DD circuit is about 50 min (Bostrom, 1974).

The third question is how the magnetic energy is released and dissipated for substorms. As a result of the current reduction in the current sheet, the "stretched" field lines must "contract."

Lui and Kamide (2003) and Akasofu (2011) suggested that electrons in the current sheet follow the contracting the field lines, but not protons, because of the breakdown of the "frozen-in" field condition. This separation process constitutes a dynamo process; in an ordinary dynamo $(\boldsymbol{V} \times \boldsymbol{B})$, protons and electrons are separated by $\pm e \boldsymbol{V} \times \boldsymbol{B}$ force. In considering the break down of the "frozen-in" field condition here, it may be recalled that in as early as 1967, Alfvén (1967, p. 440) noted in his paper titled "The second approach to cosmical electrodynamics" that "[...] the first new principle is associated with 'thaw' of the frozen-in field lines."

As a result, this dynamo produces an earthward electric field and when it is communicated to the ionosphere, it becomes an equatorward electric field that drives both the Pedersen current and the Hall current, as shown by Bostrom's 
current system (Fig. 1). In Fig. 5, the expansion phase circuit includes the substorm (UL) dynamo within the DD circuit.

In the above consideration based on Figs. 3 and 4, the required conditions are:

1. a reduction of the current in the current sheet caused by plasma instabilities, and

2. the breakdown of the "frozen-in" field condition.

Recently, Lui (2011) showed that when a substorm onset was observed on the ground on 28 February 2009, a satellite at a distance of $8.1 R_{\mathrm{E}}$ observed simultaneously:

1. plasma instabilities,

2. current reduction of about $12 \%$ in the current sheet,

3. the breakdown of the "frozen-in" field condition, and

4. the intensity of the observed earthward-directed electric field was about $10 \mathrm{mV} \mathrm{m}^{-1}$.

Thus, combining the inferred processes in the above and the observation by Lui (2011), it is possible to infer that the current reduction in the current sheet generated an earthward electric field. In addition to Lui's complete set of observations, there are several other observations in which the current reduction occurred within $10 R_{\mathrm{E}}$. The current in the current sheet that started at $7 R_{\mathrm{E}}$ and propagated outward was observed by Jacquey et al. (1991) and also by Jacquey et al. (1993) at $6 \sim 9 R_{\mathrm{E}}$. Donovan et al. (2008) reported that the expansion phase began earthward of the four THEMIS satellites and evolved radially outward. Based on the Polar spacecraft observation, Frank and Sigwarth (2000) concluded that the onset arc is connected to the equatorial distances of 5$7 R_{\mathrm{E}}$.

Further, the observed electric field is sufficient to drive Bostrom's current system; the equatorward electric field in the electrojet $\left(20 \mathrm{mV} \mathrm{km}^{-1}\right)$ requires an earthward-directed electric field of about $1.5 \mathrm{mV} \mathrm{m}^{-1}$ at a distance of $7 \sim 8 R_{\mathrm{E}}$. The observed earthward-directed electric field $\left(10 \mathrm{mV} \mathrm{m}^{-1}\right)$ is sufficient to drive the substorm (UL) current, and thus the suggested dynamo seems to work. This is the electric field that we have been looking for for a long time (cf. Akasofu, 2003). Some substorms have no sharp onset, such as that at 21:00 UT on 18 Febrary (Fig. 4). Some of the possibilities are: (a) a successive occurrence of substorms does not allow the magnetic energy accumulation, because the ionosphere can dissipate $\varepsilon$; and (b) the absence of the plasma instabilities and of the breakdown of the "frozen-in"field condition.

The observation by Lui (2011) shows that the development of the electric field was delayed for about one minute after the ground-based observation. If this delay would be confirmed by future observations, it may be that signals of the current reduction in the current sheet might propagate to the ionosphere before the earthward electric field can reach there. In this regard, it may be noted that the initial sudden brightening lasts for a few minutes and tends to occur at 22:00 23:00 MLT, where the two cells of the DD current meet in the evening sector (Fig. 2).

The fourth question is why the arcs, after the sudden brightening, advance poleward, together with the auroral electrojet. This is because the azimuthal component of Bostrom's current system (Fig. 1) produces a positive $B_{\mathrm{Z}}$ field of about $50 \mathrm{nT}$ (called "positive bays" in the midand low-latitudes) inside the current loop. The added flux $\left(\sim 10^{11} \mathrm{nT} \mathrm{km}^{-2}\right)$ of the current loop can advance the earthward edge of the azimuthal component by $600 \mathrm{~km}$ or so, corresponding to about $5^{\circ}$ or more in latitude, the extent of a large poleward expansion. Without this amount of flux, it is difficult to explain fully the expansion phase. The so-called "dipolarization" cannot provide such a large magnetic flux.

\subsection{Recovery phase}

As Fig. 4 shows, the intensity of DD current follows reasonably well the power $\varepsilon$ as a function of time after the expansion phase. The UL current subsides after the pulselike change, so that DD current dominates after it during the recovery phase. Thus, when $\varepsilon$ begins to subside, the magnetosphere tends to become an ordinary dissipative system (namely, $\varepsilon(t) \sim \delta(t-\tau)$ ) after the pulse-like expansion phase, namely about three hours after substorm onset. After the expansion phase, the ionospheric conductivity remains high enough (as the precipitation continues, in particular in the wide diffuse aurora surrounding the auroral oval), allowing the power can be dissipated as the Joule heat loss in the ionosphere without much accumulation of the magnetic energy in the magnetosphere so long as $\delta>\varepsilon$. The time delay $\tau$ is expected to be short. After a brief expansion phase, auroral arc segments drift equatorward in the midnight sector and the patches drift eastward along the auroral oval in the morning sector, delineating the DD current pattern (Fig. 2).

\subsection{Tests}

In order to test the above chain of thought, it is interesting to examine cases when a high DD dynamo power $\varepsilon$ is constantly maintained for more than $10 \mathrm{~h}$ or so during major geomagnetic storms. In such cases, there occur successive substorms, as shown in Fig. 6 (cf. Akasofu, 2004). Therefore, in such a situation, the magnetosphere releases repeatedly the accumulated magnetic energy when it reaches the limited amount, accumulating magnetic energy by overcoming the dissipation $(\varepsilon>\delta)$. Another (long puzzling) case is substorms that occur at about time when the IMF turns northward after the southward turning (Lyons et al., 2001); in such cases, the northward turning is likely to trigger a reduction of the current in the current sheet when plasma instabilities do not occur. 


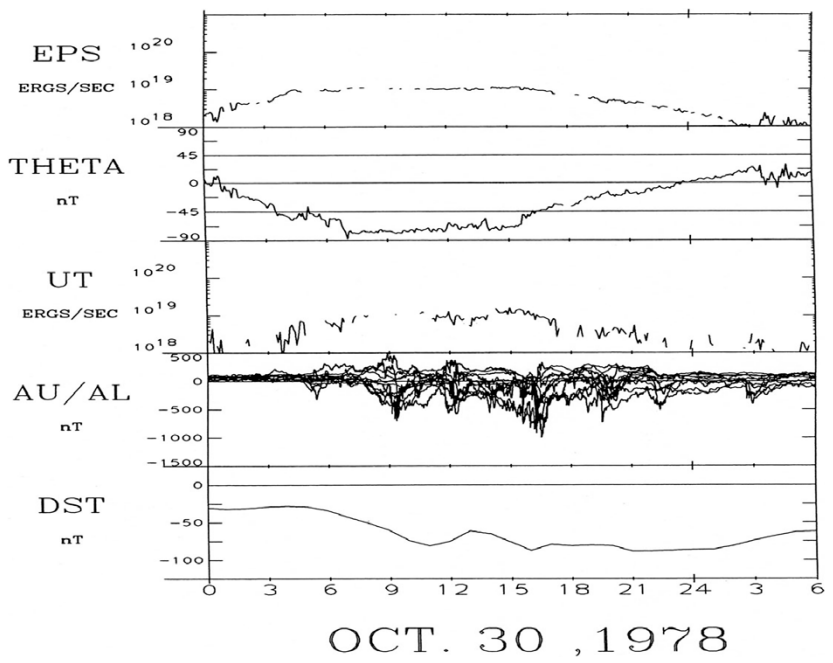

Fig. 6. An example of geomagnetic storm in which a high power $\varepsilon$ is almost constantly maintained for about $12 \mathrm{~h}$. Several substorms occurred successively during the period.

\section{Summary}

It is generally understood qualitatively that as a result of the accumulated magnetic energy during the growth phase, the magnetosphere stabilizes itself by releasing the excess energy. This paper is an attempt to make this concept a little more concrete and quantitative.

One of the unique characteristics of the magnetosphere is to release the accumulated energy like a pulse-like manner by generating the observed plasma instabilities in the current sheet and the observed current reduction. This means that both the increased amount of current in the current sheet and the "stretching" of the magnetic field lines have a limit. The release period may be controlled by the dissipation rate in the ionosphere, the time constant of the DD circuit, as well as magnetospheric conditions (the current sheet conditions, such as plasma instabilities and the "frozen-in" field). The resulting contraction of the stretched magnetic field lines establishes the observed earthward-directed electric field and drives the substorm current system that is responsible for the spectacular expansion phase of auroral substorms. The observed breakdown of the "frozen-in" field is crucial for this process. The ionosphere seems to control the magnetospheric behavior as an important energy sink of the solar wind-magnetosphere power. As a result, both the growth phase and the recovery phase can occur.

\section{Concluding remarks}

In this paper, it is shown that the concept of electrical circuit is useful in an attempt of understanding physics of the three phases of auroral/magnetospheric substorms. We know now several physical parameters which control substorms.
Further, we now have, though only as a very crude approximation, the relationship among $\varepsilon$, DD current, UL current as a function of time. Therefore, we have one of the ways of understanding the nature, more specifically the three phases of auroral substorms, and its relation to magnetospheric substorms, and also to the magnetosphere.

The aurora is the visible guide of studying magnetospheric processes. For each phase, there are many fascinating features of auroral substorms, such as the poleward expansion, westward traveling surges in the evening sector and the disintegration of the arc structure into drifting "patches" in the morning sector. Each phenomenon must be telling us about physics involved in the magnetosphere-ionosphere coupling processes. From such a point of view, a number of questions are listed in Akasofu (2012).

Acknowledgements. I would like to thank everyone, past and present, who has participated in a study of auroral substorms. I would like to thank especially Y. Kamide, W. Sun and T. Lui who worked very close with me on substorm issues.

Topical Editor I. A. Daglis thanks S. Machida and one anonymous referee for their help in evaluating this paper.

\section{References}

Akasofu, S.-I.: The development of the auroral substorm, Planet Space Sci., 12, 273-282, 1964.

Akasofu, I.-I.: Energy coupling between the solar wind and the magnetosphere, Space Sci. Rev., 28, 121-190, 1981.

Akasofu, S.-I.: Meridian chains of magnetometers as a powerful "remoste sensing" tool in determining electromagnetic quantities in the ionosphere on a global scale, EOS, 66, 465-466, 1985.

Akasofu, S.-I.: Confirmation of the validity of the electric current distribution determined by a ground-based magnetometer network, Geophys. J. Inst., 109, 191-196, 1992.

Akasofu, S.-I.: A source of aurorally electrons and the magnetospheric current systems, J. Geophys. Res., 108, 8006, doi:10.1029/2002JA009547, 2003.

Akasofu, S.-I.: Several 'controversial' issues on substotms, Space Sci. Rev., 113, 1-40, 2004.

Akasofu, S.-I.: The choice of the concept of magnetic field lines or of electric current lines: Alfvén medal lecture, Ann. Geophys., 29, 1215-1232, doi:10.5194/angeo-29-1215-2011, 2011.

Akasofu, S.-I.: Auroral morphology:A historical account and major auroral features during auroral substorms, 29-38, in: Auroral Phenomenology and Magnetospheric Processes, Earth and Other Planetts, edited by: Keiling, A., Donovan E., Begenal, F., and Karlsson, T., AGU Monograph 197, Washington, D.C., 2012.

Akasofu, S.-I., Lui, A. T. Y., and Meng, C.-I.: Importance of auroral features in the search for substorm onset processes, J. Geophys. Res., 115, A08218, doi:10.1029/2010JA016064, 2010.

Alfvén, H.: The second approach to cosmical electrodynamics, 439-444, in: The Birkeland Symposium on Aurora and Magnetic Storms, edited by: Egeland, A. and Holtet, J., Contre National de la Researche Scientifique, 1967.

Alfvén, H.: Electric currents in cosmic plasmas, Rev. Geophys. Space Phys., 15, 271-284, 1977. 
Birn, J., Hesse, M., and Schindler, K.: Formation of thin current sheets in space plasmas, J. Geophys. Res., 103, 6843-6852, 1998.

Bristow, W. A. and Jensen, P.: A superposed epoch study of Super DARN convection observations during suubstorms, J. Geophys. Res., 112, A06232, doi:10.1029/2006JA012049, 2007.

Bostrom, R.: A model of the auroral electrojet, J. Geophys. Res., 69, 4983-4999, 1964.

Bostrom, R.: Ionosphere-magnetosphere coupling, 45-59, in: Magnetospheric Physics, edited by: McCormac, B. M., Dordrecht, D. Reidel-Holland Pub. Co., 1974.

Chapman, S.: An outline of a theory ofmagnetic storms, Proc. Roy. Soc., 97, 61-83, 1918.

Chapman, S. and Ferrao, V. C. A.: The geomagnetic ring current, I. its radial stability, Terr. Mag., 46, 1-6, 1941.

Donovan, E., Liu, W., Liang, J., Spanswick, E., Voronkov, I., Connors, M., Syrjäsuo, M., Baker, G., Jackel, B., Trondsen, T., Greffen, M., Angelopoulos, V., Russell, C. T., Mende, S. B., Frey, H. U., Keiling, A., Carlson, C. W., McFadden, J. P., Glassmeier, K.H., Auster, U., Hayashi, K., Sakaguchi, K., Shiokawa, K., Wild, J. A., and Rae, I. J.: Simultaneous THEMIS in situ and auroral observations of a small substorm, Geophys. Res. Lett., 35, L17S18, doi:10.1029/2008GL033794, 2008.

Frank, L. A. and Craven, J. D.: Imaging results from Dynamic Explorer 1, Rev. Geophys., 26, 240-283, 1988.

Frank, L. A. and Sigwarth, J. B.: Finding concerning the positions of substorm onsets with auroral images from the Polar spacecraft, J. Geophys. Res., 105, 12747-12761, 2000.

Horton, W. and Doxas, I.: A low-dimensional energy-conserving state space model for substorm dynamics, J. Geophys. Res., 101, 27223-27237, 1996.
Jacquey, C., Sauvaud, J. A., and Dandouras, J.: Location and propagation of the magnetotail current disruption during substorm expansion: Analysis and simulation of an ISEE multi-onset event, Geophys. Res. Lett., 18, 389-392, 1991.

Jacquey, C., Sauvaud, J. A., and Dandouras, J.: Tailward propagating cross-tail current disruption and dynamics of near-earth tail: a multi-point measurement analysis, Geophys. Res. Lett., 20, 983 986, 1993.

Kamide, Y., Ahn, B.-H., Akasofu, S.-I., Baumjohann, W., FriisChristensen, E., Kroehl, H., Maurer, A., Richmond, A. D., Rostoker, G., Spiro, R. W., Walker, J., and Zeizev, A.: Globall distribution of ionospheric and field-aligned currents during substorms as determined from six IMS meridian chains of magnetometers: Initial results, J. Geophys. Res., 87, 8228-8240, 1982.

Lui, A. T. Y.: Reduction of the cross-tail current during near-Earth depolarization with multisatellite observations. J. Geophys. Res., 116, A12239, doi:10.1029/2011JA017107, 2011.

Lui, A. T. Y. and Kamide, Y.: A fresh perspective of the substorm current system and its dynamo, Geophys. Res. Lett., 30, 1958, doi:10.1029/2003GL017835, 2003.

Lyons, L. R., McPherron, R. L., Zesta, E., Reeves, G. D., Sigwarth, J. B., and Frank, L. A.: Timing of substormm signatures during the November 24, 1996, Geospace Environment Modeling Event, J. Geophys. Res., 106, 349-359, 2001.

Sun, W., Xu, S.-Y., and Akasofu, S.-I.: Mathematical separation of directly-driven and unloading components in the ionospheric equivalent currents during substorms, J. Geophys. Res., 103, 11695-11700, 1998. 